\title{
Occult cardiac myxoma with glandular structure detected by transesophageal echocardiography in a patient with cerebral infarction
}

\author{
Yunqi Liu ${ }^{1}$, Yanqiu Liu ${ }^{1}$, Yang Peng ${ }^{1}$, fengjuan yao ${ }^{2}$, Xiangmin $\mathrm{Li}^{1}$, and Mai Xiong ${ }^{1}$ \\ ${ }^{1}$ Sun Yat-sen University First Affiliated Hospital \\ ${ }^{2}$ Affiliation not available
}

July 16, 2020

\begin{abstract}
A previously healthy 68-year-old woman with abrupt weakness in her left limbs underwent transthoracic echocardiography for paroxysmal atrial fibrillation, which showed normal left ventricular function and no abnormalities except for left atrial dilation. However, transesophageal echocardiogram showed two leaflet-shaped loose left atrial masses were attached to the atrial septum near the oval fossa. Further CDFI found a few small vessels at the base, confirming the atrial myxoma. Surgery was performed to resect the intracardiac mass, and the histologic examination showed cardiac myxoma with glandular structure.
\end{abstract}

\section{INTRODUCTION}

Myxoma is the most common type of primary cardiac tumor in adults [1]. About $75 \%$ of cardiac myxoma originates in the left atrium, and only a few are located in the right atrium or ventricles [2]. The fragile and highly mobile myxoma can result in serious systemic embolization and left ventricular inflow tract obstruction. Echocardiography plays an important role in the diagnosis of cardiac myxomas. However, transthoracic echocardiography may be missed or misdiagnosed of small myxoma, especially in patients with risk factors for cardiac thrombosis, and it may be difficult to distinguish small myxoma from atrial thrombosis.

\section{CASE REPORT}

A previously healthy 68 -year-old woman presented to our hospital with weakness in her left limbs for 8 days. Her past medical history was unremarkable for cardiovascular disease or risk factors. On admission, she was conscious. Her blood pressure was $120 / 70 \mathrm{mmHg}$. Her cardiac auscultation revealed regular rhythm, with a heart rate $83 \mathrm{bpm}$, and no abnormal heart murmur was heard. The abdomen was soft with no mass or pain on palpation. Detailed neurologic assessment revealed weakness in her left limbs and a positive Barre' test of her left upper extremity. Brain magnetic resonance imaging showed lacunar cerebral infarction (right cerebral hemisphere), consistent with his weakness in the left extremities. Laboratory test: erythrocyte sedimentation rate (ESR) $115 \mathrm{~mm} / \mathrm{h}$ (reference value: [?]40mm/h), D dimer 1.69mg/LFEU (reference value: $0.00-0.55 \mathrm{mg} / \mathrm{LFEU}$ ), fibrinogen $6.14 \mathrm{~g} / \mathrm{L}$ (reference value: $2.00-4.00 \mathrm{~g} / \mathrm{L}$ ). ECG was performed, paroxysmal atrial fibrillation was observed once (Figure 1A). The initial transthoracic echocardiography showed normal left ventricular function and no abnormalities except for left atrial dilation (Figure 1B). We suspected cardiogenic embolism as the cause of cerebral infarction and performed a transesophageal echocardiogram (TEE). TEE showed two lobulated loosen left atrial masses measuring $20 \mathrm{~mm} \times 10 \mathrm{~mm}, 11 \mathrm{~mm} \times 8 \mathrm{~mm}$, adherent to the atrial septum near the oval fossa, with a wide base for $10 \mathrm{~mm}$, CDFI found a few small vessels at the base, the tumor had a great activity and deformability, which strongly confirmed an atrial myxoma (Figure 
2A-B). In addition, Cardiac MRI was performed: an abnormal nodular signal adherent to the left side of atrial septum, with a size of about $14 \mathrm{~mm} \times 13 \mathrm{~mm} \times 20 \mathrm{~mm}$, the lesion showed isointense signal on T1WI and inhomogeneous slightly hyperintense signal on T2WI, speckled or string like enhancement was found on first pass perfusion, enhanced and delayed reinforcement imaging compared with normal myocardium (Figure 3A-E), thus cardiac tumor a myxoma was considered. Because the cardiac tumor detected by TEE and cardiac MRI was considered to the cause of cerebral infarction, the patient was taken to the operating room for a cardiac surgery via median sternotomy under cardiopulmonary bypass, the cardiac tumor resection was successfully performed, followed by atrial septal defect repair using a pericardial patch. Postoperative TEE showed that the mass disappeared, and the atrial septal repair patch was satisfactory without atrium level shunt. On macroscopic examination, the excised tumor was $45 \mathrm{~mm} \times 25 \mathrm{~mm}$, focal papillary surface, reddish brown and jellylike (Figure 4A). Histologic examination showed the scattered or small clusters of short spindle cells and stellate cells, with glandular structure well-formed by mucous columnar epithelium in myxomatous stroma, no atypia was identified, these findings were consistent with cardiac myxoma with glandular structure (Figure 4B). With careful treatment and care, her neurological symptoms had been improved and she was discharged at the 10th day after operations without any complication.

\section{DISCUSSION}

Echocardiography plays a very important role in the diagnosis for patients with cardiac myxomas. However, for small left atrial myxoma, transthoracic echocardiography may be missed or misdiagnosed, especially in patients with risk factors for cardiac thrombosis, and it may be difficult to distinguish small early myxoma from atrial thrombosis [3]. For our patient in this case, the ultrasound imaging was so poor that we missed the tumor for the first time by transthoracic echocardiography. TEE can make up for the deficiency of transthoracic echocardiography and provide more accurate and detailed information for further diagnosing. With great high spatial resolution, characteristics of myxoma such as activity and deformation, with narrow or wide base, even small vessels in the tumor can be shown by TEE clearly, TEE is more useful in identifying cardiac tumor from thrombi compared with cardiac MRI [4]. Therefore, TEE should be routinely performed in patients who have occurred cerebral infarction in the cerebral cortex without arterial stenosis. Cardiac myxoma with glandular structure $[5,6]$ is more common in women, mostly in the left atrium, patients presented with cardiac-related or embolization symptoms. It may originate from pluripotent primitive mesenchymal cells, and pathological diagnosis should be differentiated from metastatic adenocarcinoma. The treatment of choice for benign cardiac myxoma or glandular cardiac myxoma is complete surgical excision with excellent prognosis, However, intracardiac recurrence has been noted in 12\%-22\% of familial and $1 \%-4 \%$ of sporadic cases $[3,4]$, thus careful follow-up is necessary, TEE will be very helpful especially in patients with cerebral infarction recurrence after tumor resection.

\section{CONCLUSIONS}

TEE can make up for the deficiency of transthoracic echocardiography and provide more accurate and detailed information for intracardiac mass diagnosing. Therefore, TEE should be routinely performed in patients who have occurred cerebral infarction without arterial stenosis, especially in patients with risk factors for cardiac thrombosis.

\section{CONFLICT OF INTEREST}

All authors declare that they have no conflict of interest.

\section{Reference:}

1. Dhawan S, Tak T. Left Atrial Mass: Thrombus Mimicking Myxoma. Echocardiography. 2004;21:621-3. doi: 10.1111/j.0742-2822.2004.03135.x.

2. Bernatchez J, Gaudreault V, Vincent G, et al. Left Atrial Myxoma Presenting as an Embolic Shower: A Case Report and Review of Literature. Ann Vasc Surg. 2018;53:266.e13-266.e20. doi: 10.1016/j.avsg.2018.04.024. 
3. Saito Y, Aizawa Y, Monno K, et al. Small, smooth, nonmobile cardiac myxoma detected by transesophageal echocardiography following recurrent cerebral infarction: a case report. J Med Case Rep. 2017;11:131. doi: 10.1186/s13256-017-1298-z.

4. Colin GC, Gerber BL, Amzulescu M, et al. Cardiac myxoma: a contemporary multimodality imaging review. Int J Cardiovasc Imaging. 2018;34:1789-1808. doi: 10.1007/s10554-018-1396-z.

5. Bruce CJ. Cardiac tumours: diagnosis and management. Heart. 2011;97:151-60. doi: 10.1136/hrt.2009.186320.

6. Reber D, Birnbaum DE. Recurrent cardiac myxoma: why it occurs. A case report with literature review. J Cardiovasc Surg (Torino). 2001;42:345-8.

Figure 1 A. ECG showed, paroxysmal atrial fibrillation was observed once. B. The first transthoracic echocardiography showed no abnormalities except for left atrial dilation. RA: right atrium; RV: right ventricle; LA: left atrium.

Figure 2 Transesophageal echocardiography (TEE) showed: A. a lobulated loosen mass in the left atrium, adherent to the oval fossa, measuring $20 \mathrm{~mm} \times 10 \mathrm{~mm}, 11 \times 8 \mathrm{~mm}$, with a wide base for $10 \mathrm{~mm}$ (yellow arrow); B. CDFI showed a few small vessels at the base of the tumor (yellow arrow). RA: right atrium; LA: left atrium.

Figure 3 Cardiac magnetic resonance imaging (MRI) showed: A-B. a left atrial mass measuring $14 \mathrm{~mm} \times 13 \mathrm{~mm} \times 20 \mathrm{~mm}$ adherent to the fossa ovalis. C. The lesion showed enhancement on first pass perfusion, early enhanced (3.5min, D) and delayed (10min, E) reinforcement imaging compared with normal myocardium (yellow arrow). RA: right atrium; RV: right ventricle; LA: left atrium; LV: left ventricle; PA: pulmonary artery; aAO: ascending aorta; dAO: descending aorta.

Figure $4 \mathrm{~A}$. the excised tumor was $45 \mathrm{~mm} \times 25 \mathrm{~mm}$, focal papillary surface, reddish brown and jellylike. B. Histologic examination showed the scattered or small clusters of short spindle cells and stellate cells, with glandular structure formed by mucous columnar epithelium in myxoid background, no atypia (H\&E, x100). 
A
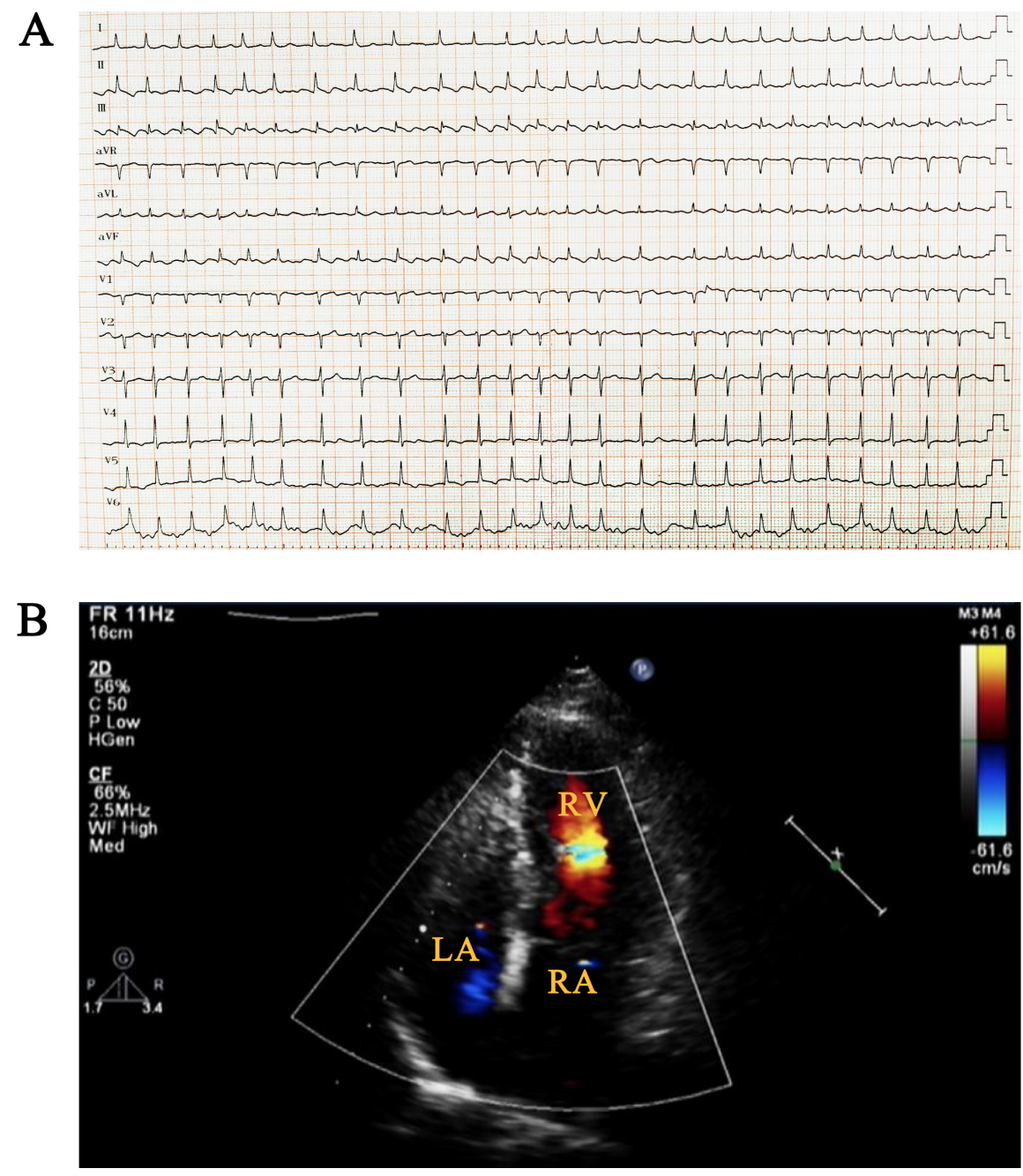

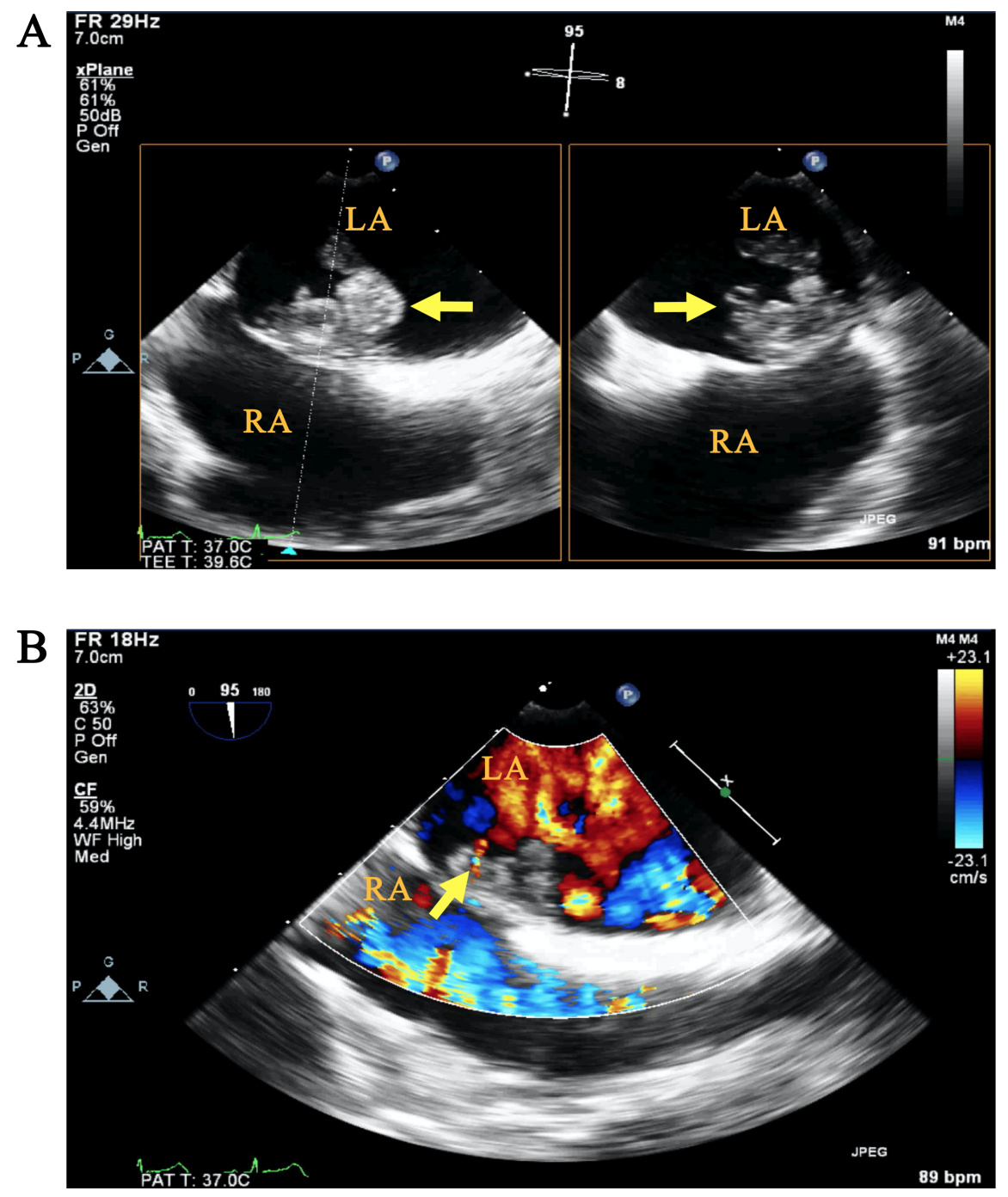


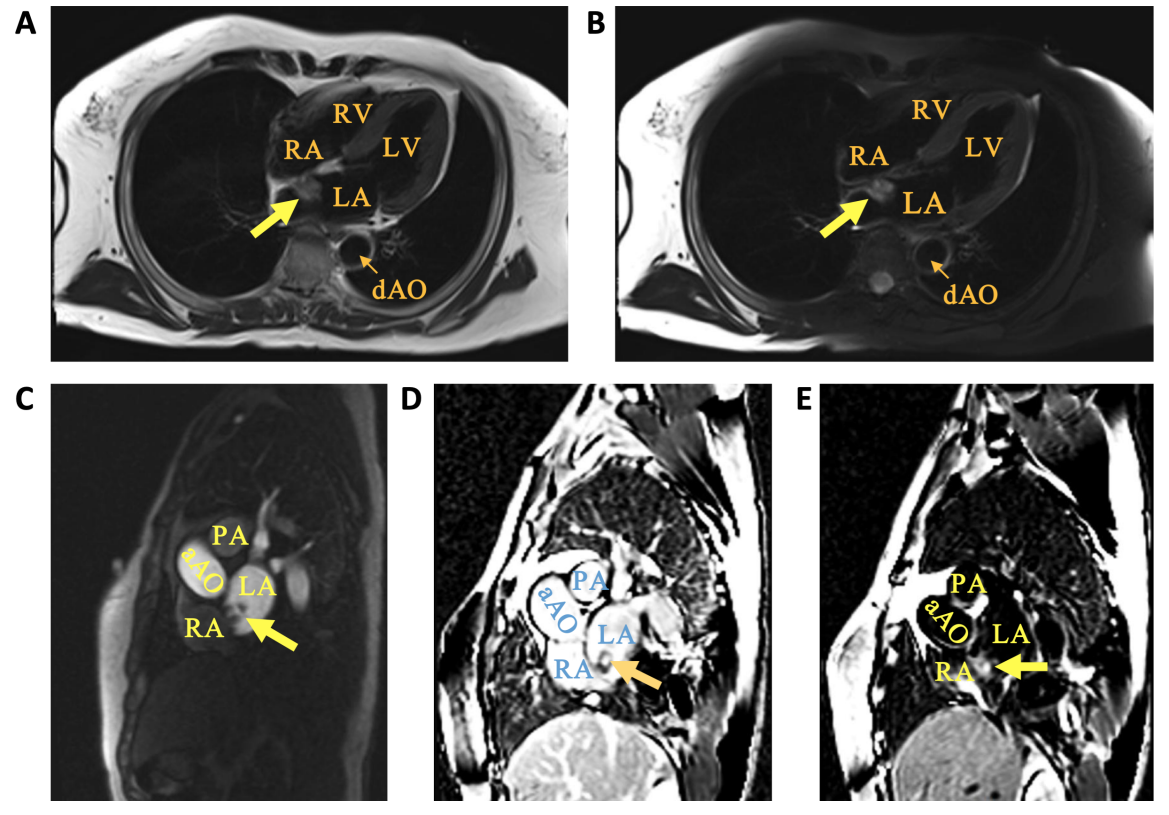



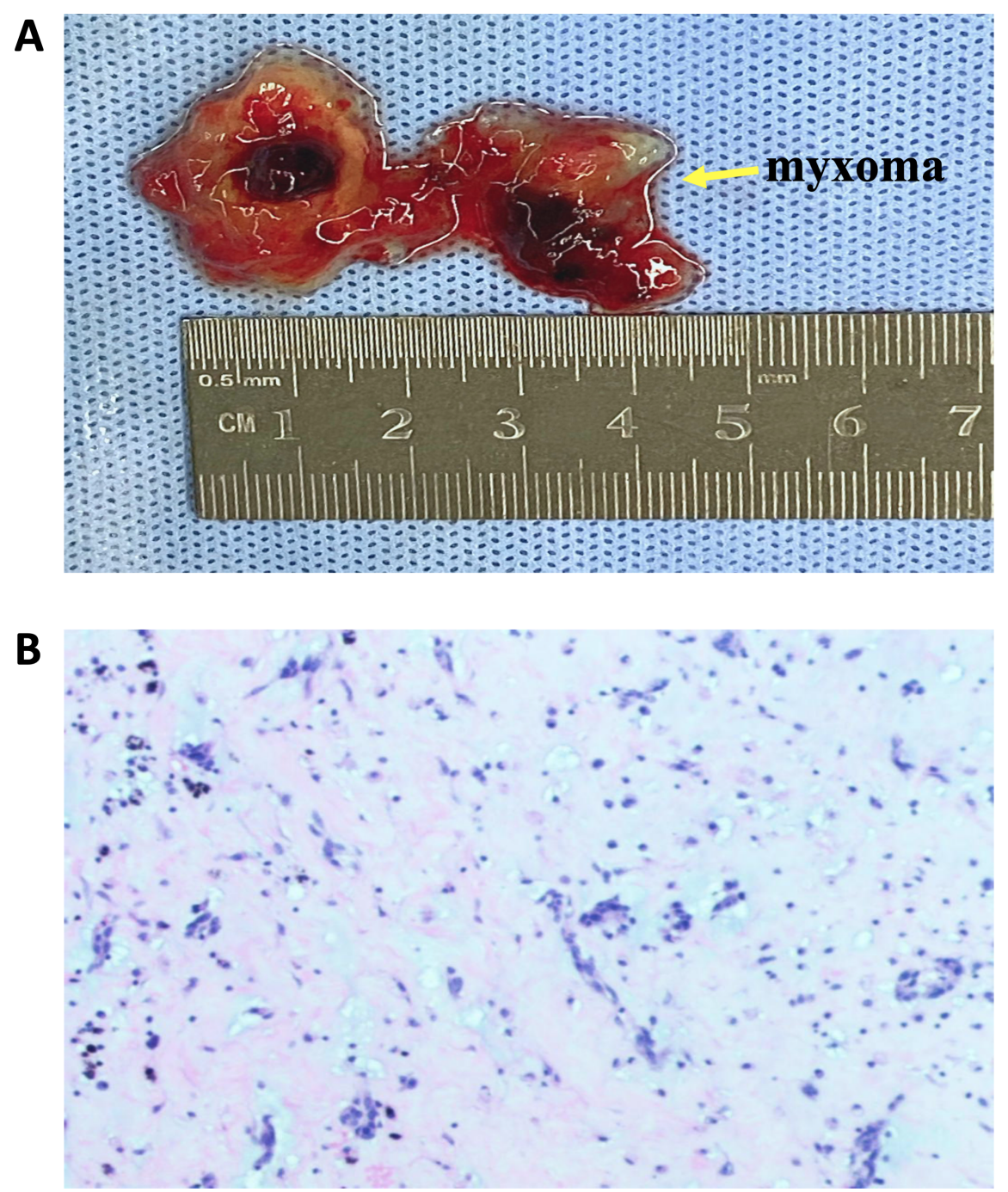